\title{
T300 Carbon/Epoxy Torsion Bar as a Replacement to Grade 5 Titanium in Formula 1 Using Finite Element Analysis
}

\author{
K. I. Ahmad' ${ }^{1}$ M. A. Kariem², J. L. Curiel-Sosa ${ }^{3}$ \\ ${ }^{1}$ Mechanical Engineering Undergraduate Program \\ Faculty of Mechanical and Aerospace Engineering Institut Teknologi Bandung, Bandung, Indonesia \\ ${ }^{2}$ Engineering Design Research Group, Faculty of Mechanical and Aerospace Engineering, Institute \\ Technology Bandung, Bandung, Indonesia \\ ${ }^{3}$ Computer-Aided Aerospace and Mechanical Engineering Group Department of Mechanical Engineering the \\ University of Sheffield, Sheffield, United Kingdom
}

\begin{abstract}
In this paper, an analysis of a carbon fibre reinforced plastic (CFRP) torsion bar in a Formula 1 car is discussed. The use of composite torsion bar is rather uncommon on in the application of Formula 1 car. Composite structures are usually applied to produce a lighter design while maintaining the strength. Composite structures are considered as modern materials that may act as an upgrade to the conventional metal alloys in present engineering applications. In a Formula 1 car, the load applied on a torsion bar could cause fatigue due to its frequent usage. Maintaining safety and weight are crucial for Formula 1 cars. Replacing metal components with composite could be beneficial. Torsion bars are vital components on a Formula 1 car's suspension. The torsion bar acts as a spring to absorb bumps by utilizing its stiffness when it experiences torsion. A comparison between the stresses of the torsion bar for both composite and metal alloy is conducted in the present work. This is done by simulating a torsion test on a torsion bar model with predetermined conditions. The comparison of the torsion test simulation results the for both materials by means of Finite Element Method (FEA) is shown in some details.
\end{abstract}

Keywords: composite structures; finite element method; stress concentration; torsion bar; torsional test 


\section{Introduction}

Suspensions are integral components in vehicles. Its main function is to improve a vehicle's ride and handling. The vehicle's steering cooperates with the suspension and tires to ensure a smooth ride and steering. The suspension has three key components to accomplish the goals above, which are a frame, springs, and damper. The spring component of a Formula 1 car take two forms of setup; torsion bar setup and coil spring setup. Coil spring setups are the more traditional suspension system and they are frequently found in modern cars.

The torsion bar has the same task as a coil spring but is more compact. These suspension systems are mounted on the chassis above and in front of the driver's legs, the front of the car, and on top of the gearbox at the rear of the car. Torsion bar takes the shape of a tube or bar, usually made out of metal. Torsion bars are now in use on all Formula 1 cars due to its compact trait. The performance of the torsion bar depends on its stiffness when it is twisted. As the torsion bar absorbs a bump, it will twist in a direction and will return to its initial shape by twisting back to the other direction (Formula 1 Dictionary, 2020).

In competitive racing, performance is always optimised to achieve the best time possible for a car to finish the race. Composites are believed to surpass the performance of metal alloys. They exhibit a comparable strength to metal alloys yet they possess much lower densities. Thus, fibre reinforced composites boast improved properties such as strength and stiffness per unit weight. Substituting metal alloy components with composite components would enable Formula 1 cars to have reduced weight, hence improving the overall performance of the car during racing (Savage, 2008).

The torsion experienced by the torsion bar would result in stress. The stress causes the torsion bar to deform as it twists in the same direction of the torsion. The maximum shear stress due to torsion may be defined as the product of torsion and radius over the polar moment of inertia.

$$
\tau=\frac{T C}{J}
$$

The polar moment of inertia of a tube can be defined using Eq. 2 below.

$$
\pi\left(c_{o}^{4}-c_{i}^{4}\right)
$$

$$
J=
$$

The deformation of the torsion bar can be represented by the angle of twist. The angle of twist is defined as the ratio between the product of torsion and length with the product of polar moment of inertia and the shear modulus of the material (Hibbeler, 2011).

$$
\theta=\frac{T L}{J G}
$$




\subsection{Assumptions}

To simplify the analysis of the torsion bar model, a few assumptions are taken into consideration. The assumptions are:

a) Straight bar and uniform cross section;

b) Torsional load only;

\section{Structural Integrity Analysis of CFPR and Titanium Alloy Torsion Bar To} compare a composite and a metal alloy torsion bar, Simulia ABAQUS software is used to analyse the stress concentrations on a carbon fibre reinforced plastic (CFPR) torsion bar and a titanium alloy torsion bar by utilizing finite element analysis. The torsion bar models are selected to be made up of unidirectional T300 carbon fibre/epoxy composite and annealed grade 5 titanium (Ti-6Al-4V) respectively. The properties of the respective materials are shown below.

Table 1: T300 Carbon Fibre/Epoxy Composite Properties

\begin{tabular}{|l|c|}
\hline \multicolumn{1}{|c|}{ Property } & Value \\
\hline Longitudinal Modulus, $\mathrm{E}_{11}(\mathrm{GPa})$ & 133.38 \\
\hline Transverse Modulus, $\mathrm{E}_{22}=\mathrm{E}_{33}(\mathrm{GPa})$ & 8.29 \\
\hline Poisson's Ratio, v12=V13 & 0.26 \\
\hline Poisson's Ratio, v23 & 0.424 \\
\hline Inplane Shear Modulus, $\mathrm{G}_{12}=\mathrm{G}_{13}(\mathrm{GPa})$ & 3.81 \\
\hline Transverse Shear Modulus, $\mathrm{G}_{23}(\mathrm{GPa})$ & 2.9 \\
\hline
\end{tabular}

Source: (Sinha, 2006)

Table 2: Annealed Grade 5 Titanium Alloy Properties

\begin{tabular}{|l|c|}
\hline \multicolumn{1}{|c|}{ Property } & Value \\
\hline Density $\left(\mathrm{g} / \mathrm{cm}^{3}\right)$ & 4.43 \\
\hline Modulus of Elasticity $(\mathrm{GPa})$ & 113.8 \\
\hline Poisson's Ratio & 0.342 \\
\hline
\end{tabular}

Source: (ASM Material Datasheet, 2020)

The model designed for simulation is shown in Fig. 1(a) and Fig. 1(b) where the model is shaped as a thick-walled tube with splined ends.

Figure 1(a) Torsion Bar Model Isometric View

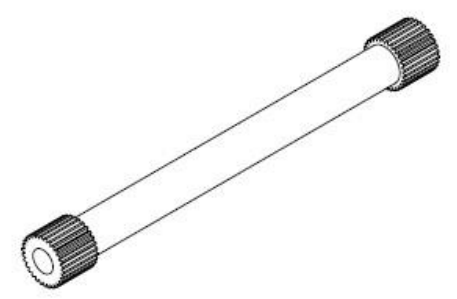




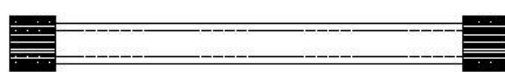

\subsection{Grade 5 Titanium Torsion Bar Torsional Test Simulation}

The torsion test is done by simulating a torsional load on the rotating end at $200 \mathrm{Nm}$ counterclockwise on the longitudinal axis. This is about equal to the typical weight of Formula 1 cars multiplied with the outer diameter on the grade 5 titanium torsion bar. Since the torsion bar is symmetrical along the longitudinal axis the model is sectioned half its length. The result of the simulation of the grade 5 titanium torsion bar is shown in Fig. 2(a) and Fig. 2(b).

Figure 2(a): Grade 5 Titanium Torsion Bar Stress Distribution



Figure 2(b): Maximum Stress Location

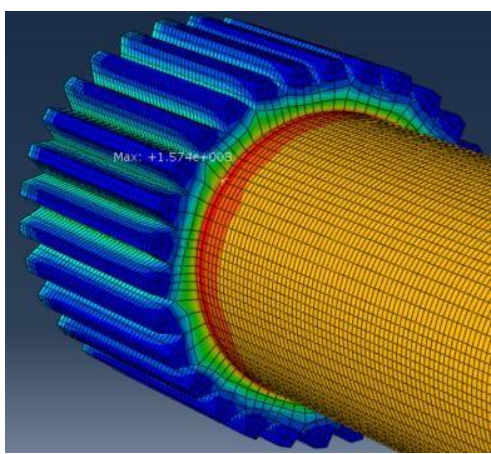

The results above shows that the splined ends experience less stress than the tube since the cross section of the splined ends have greater surface area. The stress is concentrated on the edge where the tube and the splined end meet. At this point, the difference in diameter causes the shear stress to be greater as shown in the definition of polar moment of inertia in Eq. 2 and its relationship with stress in Eq. 1. The stress is maximum where the tube meets the rotating end at a value of 157.4 MPa. This is due to the relationship between the stress and the radius shown in Eq. 1 where they are directly proportional or in other words, stress will be maximum at the outer diameter. 


\subsection{T300 Carbon Fibre/Epoxy Torsion Bar Torsional Test Simulation}

The composite torsion bar is tested with the same conditions as the titanium alloy torsion bar. The composite torsion bar is designed with a unidirectional where the composite is plied in the radial direction.

Figure 3(a): T300 Carbon/Epoxy Composite Torsion Bar Stress Distribution

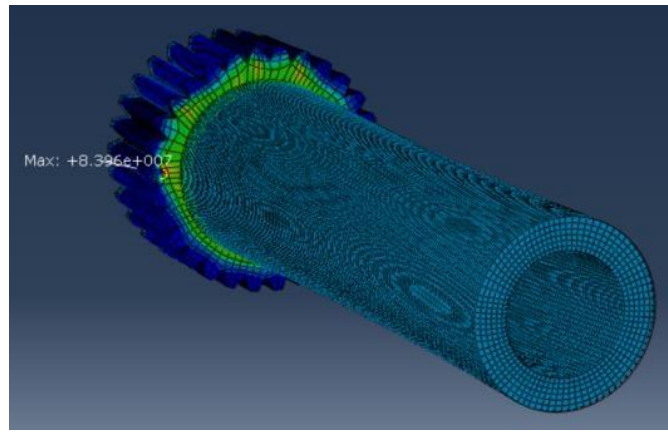

Figure 3(b): Maximum Stress Location

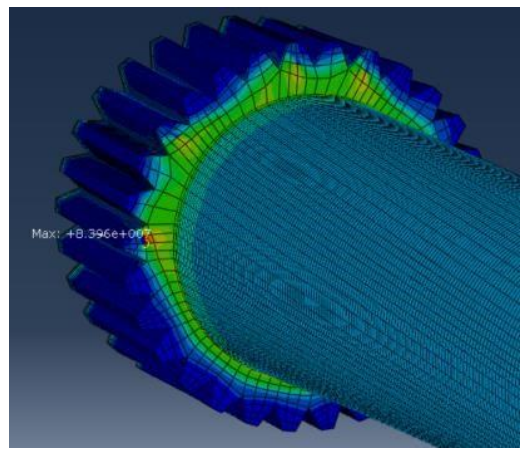

The maximum stress is shown to be near the outer diameter of the splined end, just before the splined region. This also is related to Eq. 1 and since the ridges also have a small crosssectional area in along the longitudinal axis, a greater stress concentration is produced. The composite torsion bar has a significantly lower maximum stress value at $83.96 \mathrm{MPa}$ and has less stress concentrated on the tube region.

\section{Conclusion}

The T300 carbon fibre/epoxy composite torsion bar has a safer stress concentration in comparison to the conventional grade 5 titanium torsion bar. The maximum stress on the composite torsion bar is nearly half the stress on the titanium torsion bar. In addition to that, there are more critical regions on the titanium torsion bar whereas the critical area on the composite torsion bar is mostly localized on the point where stress is maximum. It can be concluded that the composite torsion bar can provide more benefits than an alloy torsion bar. A composite torsion bar is much lighter but more importantly, is safer because its ratio between the maximum stress and the material strength is lesser.

\section{Acknowledgment}

This paper is dedicated to various individuals that assisted the author in completing the bachelor's thesis. First and foremost, the author would like to give the utmost gratitude to God, 
whom have blessed the author with a functional body, mind and soul that allowed the author to dedicate four years of knowledge to this paper. The author is also grateful towards the assistance and guidance of the supervising lecturers, M. Agus Kariem ST., MT., Ph.D. and Dr. Jose Luis Curiel-Sosa, who dedicated their time and effort which played a huge role in this paper, for without them this paper might have not existed. Gratitude is also shown to Dr. Mahesa Akbar who have voluntarily assisted the author in making this paper possible by facilitating the publishing of this paper. The author would also like to thank his loving parents, Moureen Achmad and Achmad Zailani, that supported his life both financially and emotionally since birth. Finally, the author shows his thanks as well to any other individual that is not mentioned by name.

\section{References}

[1] ASM Material Datasheet. (n.d.). $\quad$ Retrieved from http://asm.matweb.com/search/SpecificMaterial.asp?bassnum=MTP641

[2] Hibbeler, R. C., \& Yap, K. B. (2010). Mechanics of materials (8th ed.). Prentice Hall: Pearson.

[3] Hoeltgebaum, T., \& Vieira, R. D. S. (2012). A Design Comparison between Coil Springs and Torsion Bars. In A Design Comparison between Coil Springs and Torsion Bars.

[4] Kress, G. R., \& Ermanni, P. A. (n.d.). CFRP Torsion Bar: Load Introduction Problem.

[5] Pietrich, M., Schrodin, B., \& Kletzin, U. (2017). Material Selection Method for Composite Springs.

[6] Savage, G. (2008). Composite Materials Technology in Formula 1 Motor Racing. Honda Racing F1.

[7] Seas. (n.d.). Torsion bar. Retrieved from http://www.formula1-dictionary.net/torsion_bar.html [8] Sinha, P. K. (2006). Composite materials and structures. Composite Centre of Excellence.

[9] Vinchure, P. V., \& Kumbhar, S. B. (2018). Analysis of Glass Fiber Reinforced Composite Torsion Bar. International Journal of Advance Research in Science and Engnineering, 7(3). 